\title{
LIGHT EMITTING DIODE VERSUS CONVENTIONAL PHOTOTHERAPY FOR THE TREATMENT OF NEONATAL HYPERBILIRUBINEMIA: A SYSTEMATIC REVIEW AND META-ANALYSIS
}

\author{
A. Tridente ${ }^{1}$, D. de Luca $^{2}$ \\ ${ }^{I}$ Sheffield Teaching Hospitals NHS Foundation Trust, Sheffield, UK, ${ }^{2}$ Universita Cattolica, Rome, Italy
}

Background: The efficacy of phototherapy for neonatal jaundice depends on a number of factors, including the spectrum of light emitted. Various light emitting diode (LED) phototherapy devices have been trialled on the assumption of a more effective spectral distribution of the light emitted.

Objectives: We reviewed the current literature to determine whether, in jaundiced newborns, LED phototherapy is more effective than conventional phototherapy in lowering total serum bilirubin (TSB).

Data sources: The PubMed, EMBASE and Cochrane Central Register (Clinical Trials) databases were searched.

Study eligibility criteria, participants, interventions: Studies eligible for inclusion were randomized controlled trials of LED versus conventional phototherapy conducted on neonatal hyperbilirubinemia, published in peer reviewed journals.

Study appraisal and synthesis methods: Studies were found to be of medium quality based on a components approach. Results were statistically aggregated within a very homogeneous population (term or late preterm neonates) and appeared robust when sensitivity analyses were performed.

Results: LED and conventional phototherapy devices appeared to be equally effective in reducing TSB in term or late preterm neonates (pooled average difference in rate of decrease of TSB was $0.194 \mu \mathrm{mol} / \mathrm{L} / \mathrm{h}$ $[0.011 \mathrm{mg} / \mathrm{dL} / \mathrm{h}]$ in favour of LED phototherapy, $\mathrm{p}=0.378)$, and had comparable incidence of adverse events.

Limitations: The methodological quality of the studies included and the possible presence of publication bias are the main limitations.

Conclusions and Implications of key findings: Further randomized controlled trials are needed to ascertain whether LED phototherapy may be more effective when increasing the spectral power, or in certain selected subpopulations. 